\title{
Listeriosis Caused by Persistence of Listeria monocytogenes Serotype 4b Sequence Type 6 in Cheese Production Environment
}

\author{
Magdalena Nüesch-Inderbinen, Guido V. Bloemberg, Andrea Müller, \\ Marc J.A. Stevens, Nicole Cernela, Beat Kollöffel, Roger Stephan
}

A nationwide outbreak of human listeriosis in Switzerland was traced to persisting environmental contamination of a cheese dairy with Listeria monocytogenes serotype 4b, sequence type 6 , cluster type 7488 . Whole-genome sequencing was used to match clinical isolates to a cheese sample and to samples from numerous sites within the production environment.

工 isteriosis is a potentially lethal infection, and the elderly population, pregnant women, and immunocompromised persons at particular risk (1). Foods, in particular ready-to-eat foodstuffs, including meat, fish, dairy products, fruits, and vegetables, represent the major vehicle for sporadic cases and outbreaks of listeriosis (2). Listeria monocytogenes serotype $4 \mathrm{~b}$ sequence type 6 (ST6) has emerged since 1990 as a hypervirulent clone that is associated with particularly worse outcome for case-patients who have Listeria meningitis and therefore poses a particular threat to consumer health $(3,4)$.

L. monocytogenes ST6 is increasingly associated with outbreaks, including an outbreak linked to frozen vegetables in 5 countries in Europe during 20152018 (5), an outbreak associated with contaminated meat pâté in Switzerland during 2016 (6), and the largest listeriosis outbreak globally, which occurred in South Africa during 2017-2018 (7,8). More recently, the largest outbreak of listeriosis in Europe in the past 25 years was reported in Germany and was traced

Author affiliations: Institute for Food Safety and Hygiene, Zurich, Switzerland (M. Nüesch-Inderbinen, M.J.A. Stevens, N. Cernela, R. Stephan); National Reference Centre for Enteropathogenic Bacteria and Listeria, Zurich (G.V. Bloemberg, A. Müller); Kantonales Laboratorium der Urkantone, Brunnen, Switzerland (B. Kollöffel)

DOI: https://doi.org/10.3201/eid2701.203266 back to blood sausages contaminated with L. monocytogenes ST6 belonging to a particular clone referred to as Epsilon1a (9).

Human listeriosis is a reportable disease in Switzerland. All cases of culture- or PCR-confirmed human listeriosis are reported to the Swiss Federal Office of Public Health (SFOPH). Diagnostic laboratories and regional (cantonal) laboratories forward isolates to the Swiss National Reference Centre for Enteropathogenic Bacteria and Listeria for strain characterization, ensuring early recognition of Listeria clusters among food isolates or human cases. We report an outbreak of listeriosis associated with cheese contaminated with L. monocytogenes $4 \mathrm{~b}$ ST6 in Switzerland.

\section{The Study}

In 2018, the SFOPH recorded 52 human cases of listeriosis, corresponding to a normal annual incidence rate of 0.6 cases $/ 100,000$ inhabitants (10). However, during March 6, 2018-July 31, 2018, an increase of L. monocytogenes serotype $4 \mathrm{~b}$ from 13 human cases was recorded. Whole-genome sequencing (WGS) was performed on these strains by using MiSeq next generation sequencing technology (Illumina, https://www.illumina. com). Sequencing reads were mapped against an mutlilocus sequencing typing (MLST) scheme based on 7 housekeeping genes and a 1,701-locus core genome MLST (cgMLST) scheme by using Ridom SeqSphere+ software version 5.1.0 (11). STs and cluster types (CTs) were determined upon submission to the L. monocytogenes cgMLST Ridom SeqSphere+ server (http:// www.cgmlst.org/ncs/schema/690488/).

A cluster was defined as a group of isolates with $\leq 10$ different alleles between neighboring isolates $(9,11)$. Twelve of 13 isolates were assigned to ST6 CT7448, a unique profile in the database, showed 
by cluster detection to be closely related. Accordingly, we defined an outbreak case-patient as a patient who had listeriosis and L. monocytogenes ST6 CT7448. An outbreak investigation was initiated by the SFOPH, and patients were contacted to assess food exposures by using a standardized questionnaire. Diagnostic and cantonal laboratories were notified nationwide to ensure rapid submission of L. monocytogenes isolates to the National Reference Centre for Enteropathogenic Bacteria and Listeria for laboratory typing, including WGS. However, the questionnaire-based outbreak investigation did not lead to a suspect food, and the vehicle of infection remained unknown.

In a second wave, onset dates ranged from January 22 to May 26, 2020 (Figure 1). Another 27 cases of infection with L. monocytogenes serotype $4 \mathrm{~b}$ were recorded; 4 cases were in hospital patients who had underlying conditions. During this period, questionnaire-based data were not available to support a food hypothesis.

A total of 22 strains grouped on the basis of WGS in a tight cluster, with the exception of N20-2045, which differed by $\geq 8$ alleles (Figure 2). This strain was within the cluster definition. However, in absence of supportive epidemiologic data, we were not able to verify whether N20-0245 was truly involved in the outbreak.

Median age of the patients was 81 years (range $<1-99$ years). More than half of the patients were female $(18 / 34,53 \%)$. Of the 34 human isolates, 30 were from blood samples and 1 each from an abscess, ascites, maternal placenta tissue, or stool sample (Table).
One case of perinatal transmission and 10 deaths (29\%) were reported.

On April 30, 2020, a cheese manufacturer reported to the cantonal laboratory detection of L. monocytogenes from a sample of soft (brie) cheese made from pasteurized milk. Analysis had been conducted as part of the manufacturer's routine quality control practices, which are mandatory in Switzerland (Swiss Foodstuffs Act, Article 23). The cheese isolate N20639 matched the outbreak strain CT by WGS (Table; Figure 2). The cantonal authorities started the tracing of the distribution chain of the dairy. The cheese producer supplied several buyers who provide cheese to retailers throughout Switzerland. The buyers were requested to immediately stop the delivery of the products of this specific producer.

These findings prompted extensive environmental sampling on the production site of the manufacturer. A total of 50 swab specimens from locations, such as vats, cheese harps, skimming devices, sink drains, brushes, scrub sponges, trays, door handles, ripening cellar floors, and walls were obtained. Swabs were incubated in Half Frazer Broth (Bio-Rad, https:/ / www. bio-rad.com) at $30^{\circ} \mathrm{C}$ for $48 \mathrm{~h}$. L. monocytogenes was detected by real-time PCR with the Assurance Genetic Detection System (Endotell, https://www.endotell. ch) according to the manufacturer's instructions. To obtain strains for WGS, 5 enriched Half Frazer Broth cultures were streaked on chromogenic Listeria agar plates (Oxoid, Pratteln, Switzerland) and incubated at $37^{\circ} \mathrm{C}$ for $24 \mathrm{~h}$.

L. monocytogenes was identified in 11 (22\%) of 50 environmental samples, and all 5 sequenced isolates

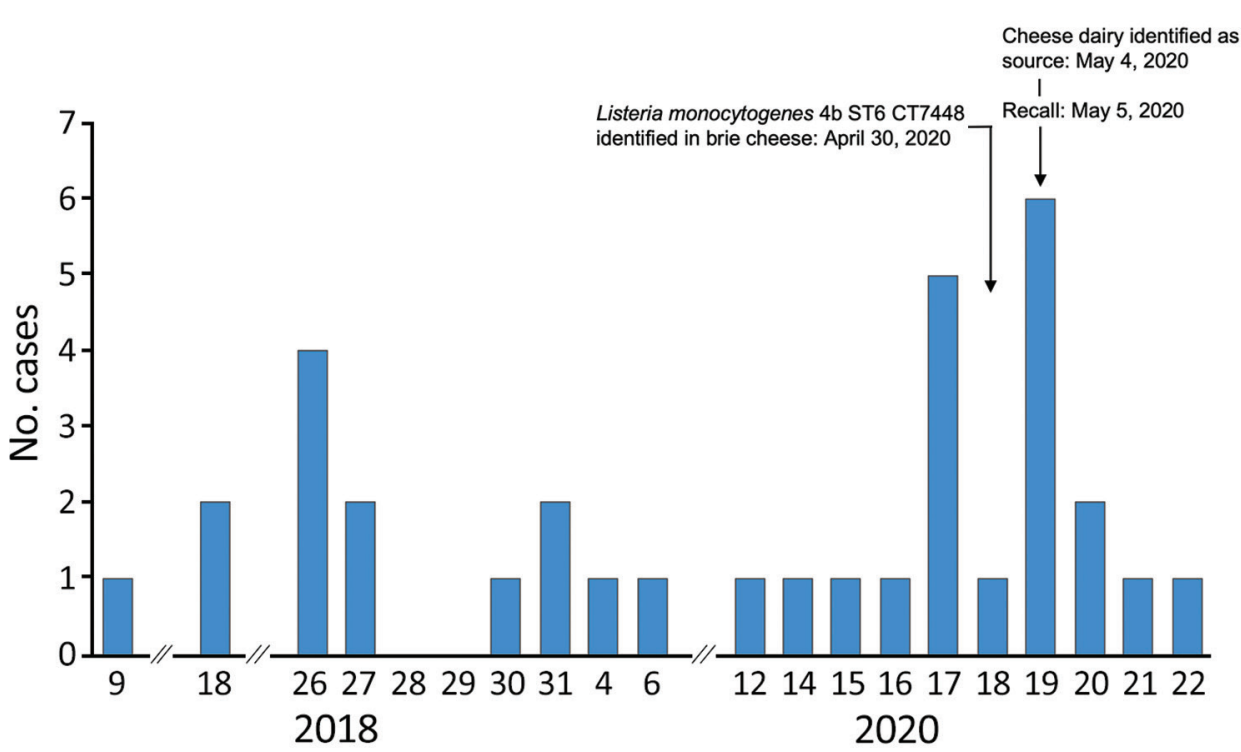

Dates of onset, week and year
Figure 1. Cases of human listeriosis caused by Listeria monocytogenes ST6 CT7488, by week and year, Switzerland, 2018 and 2020. CT, cluster type; ST, sequence type. 


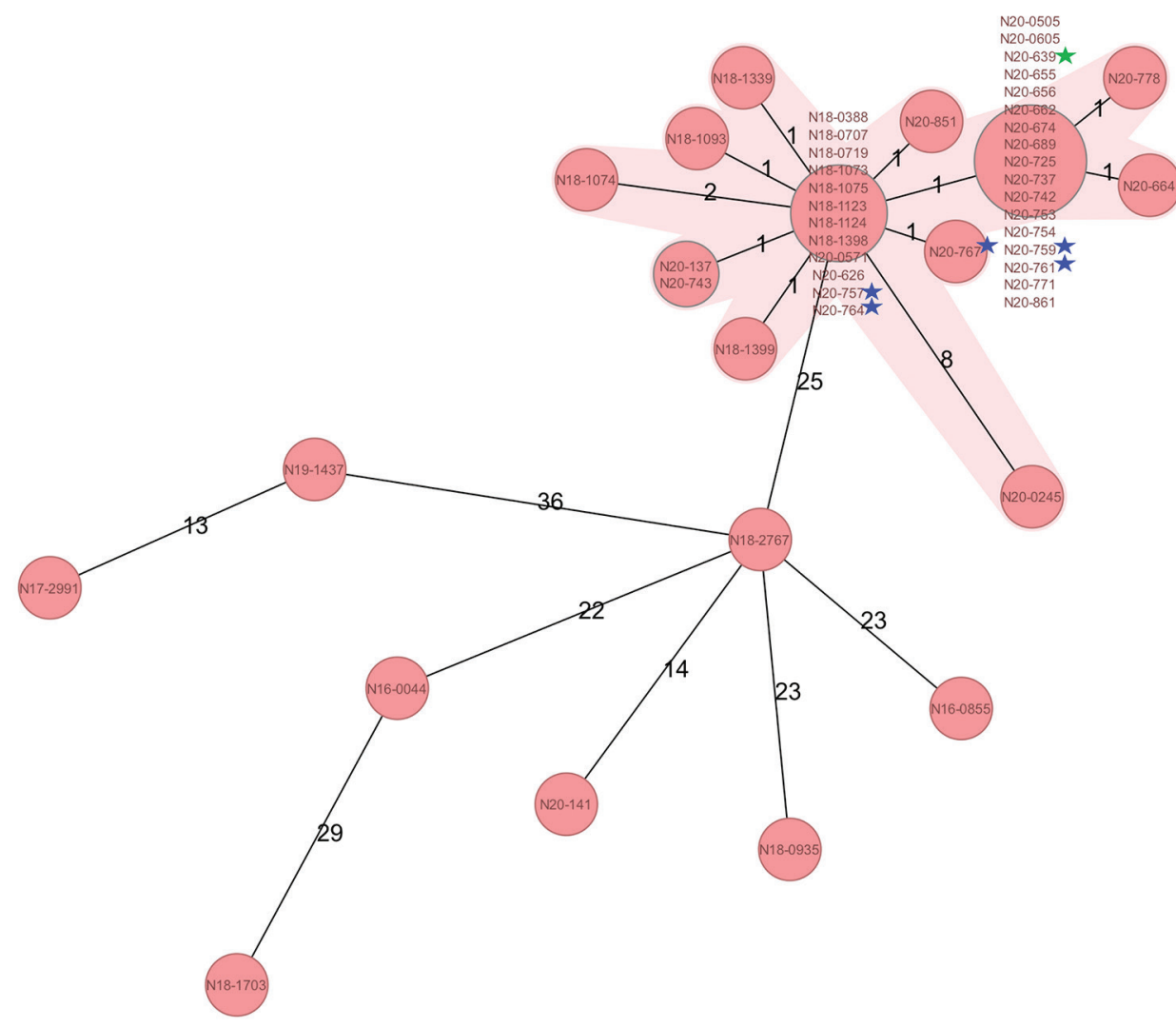

Figure 2. Minimum-spanning tree based on cgMLST allelic profiles of 34 human Listeria monocytogenes isolates, 1 food isolate, and 5 environmental isolates, Switzerland. Each circle represents an allelic profile based on sequence analysis of 1,701 cgMLST target genes. Values on connecting lines indicate number of allelic differences between 2 strains. Each circle contains the strain identification(s). The food isolate is indicated by a green star, and environmental strains are indicated by blue stars. Outbreak strains are shaded in pink and are shown in comparison with other $L$. monocytogenes sequence type 6 isolates from Switzerland collected during 2016-2020. cgMLST, core genome multilocus sequence typing.

matched the outbreak strain CT (Table; Figure 2). These results lead to a recall on May 5, 2020, of 26 items, including brie, sheep and goat cheese, and organic cheeses; production was stopped immediately. The findings were reported to the Epidemic Intelligence Information System for Food and Waterborne Diseases and Zoonoses. After the recall of the implicated products and a public warning issued by the Federal Food Safety and Veterinary Office, 7 cases of listeriosis caused by the outbreak strain were recorded (Figure 1). The last known case caused by this outbreak strain was sampled on May 20, 2020, and reported to SFOPH on May 25, 2020. Sequence data have been deposited in the National Center for Biotechnology Information (Bethesda, MD, USA) BioSample database under project no. PRJNA640586. We provide accession numbers (Table).

\section{Conclusions}

This prolonged outbreak of L. monocytogenes $4 \mathrm{~b}$ ST6 CT7448 caused 34 laboratory-confirmed listeriosis cases and 10 deaths. The outbreak investigation is an example of successful collaboration between laboratories and food safety and public health authorities to determine sources of contamination and reconstruct outbreak development. The results of the investigation implicated a cheese dairy with sanitation shortcomings and persisting environmental contamination throughout the production site. Isolation and WGS typing of L. monocytogenes from a quality-control cheese sample provided crucial information that enabled identification of the origin of contamination. WGS played a key role in showing close relatedness between the isolates from the cheese item and from the environment, and in linking the listeriosis cases from 2018 to the 2020 outbreak.

This outbreak highlights the risk for recontamination of pasteurized cheese products during manufacturing and emphasizes the need for routine sampling of products, manufacturing equipment, and the production environment. Routine quality controls should include WGS typing of environmental $L$. monocytogenes isolates to enable early recognition of potential food contamination and to ultimately mitigate the risk for listeriosis.

\section{Acknowledgments}

We thank the Swiss Federal Office of Public Health and the Federal Food Safety and Veterinary Office for critically reviewing the manuscript.

This study was partly supported by the Swiss Federal Office of Public Health, Division Communicable Diseases. 
Table. Listeria monocytogenes 4b sequence type 6 cluster type 7448 isolates associated with listeriosis outbreak, Switzerland, 20182020*

\begin{tabular}{|c|c|c|c|c|c|}
\hline Isolate ID & Date of isolation & Origin & Source & Patient age, $y /$ sex & $\begin{array}{c}\text { BioSample } \\
\text { accession no. }\end{array}$ \\
\hline N18-0388 & 2018 Mar 6 & Human & Blood & $82 / \mathrm{F}$ & SAMN15325567 \\
\hline N18-0707 & 2018 Apr 30 & Human & Ascitic fluid & $79 / F$ & SAMN15325568 \\
\hline N18-0719 & 2018 May 2 & Human & Blood & $59 / F$ & SAMN15325569 \\
\hline N18-1073 & 2018 Jun 26 & Human & Blood & $<1 / F$ & SAMN15325570 \\
\hline N18-1074 & 2018 Jun 26 & Human & Blood & $88 / F$ & SAMN15325571 \\
\hline N18-1075 & 2018 Jun 26 & Human & Maternal placenta tissue & $38 / \mathrm{F}$ & SAMN15325572 \\
\hline N18-1093 & 2018 Jun 27 & Human & Blood & $82 / \mathrm{M}$ & SAMN15325573 \\
\hline N18-1123 & 2018 Jul 3 & Human & Blood & $81 / \mathrm{M}$ & SAMN15325574 \\
\hline N18-1124 & 2018 Jul 3 & Human & Blood & 99/M & SAMN15325575 \\
\hline N18-1339 & 2018 Jul 24 & Human & Blood & $82 / F$ & SAMN15325576 \\
\hline N18-1398 & 2018 Jul 31 & Human & Blood & 48/M & SAMN15325577 \\
\hline N18-1399 & 2018 Jul 31 & Human & Blood & 14/M & SAMN15325578 \\
\hline N20-0137 & 2020 Jan 22 & Human & Blood & $77 / \mathrm{M}$ & SAMN15325579 \\
\hline N20-0245 & 2020 Feb 7 & Human & Blood & 73/M & SAMN15325580 \\
\hline N20-0505 & 2020 Mar 17 & Human & Blood & 73/M & SAMN15325581 \\
\hline N20-0571 & 2020 Mar 30 & Human & Blood & $85 / \mathrm{M}$ & SAMN15325582 \\
\hline N20-0605 & 2020 Apr 6 & Human & Blood & $73 / \mathrm{M}$ & SAMN15325583 \\
\hline N20-0626 & 2020 Apr 15 & Human & Blood & $85 / \mathrm{M}$ & SAMN15325584 \\
\hline N20-0655 & 2020 Apr 20 & Human & Blood & $66 / \mathrm{F}$ & SAMN15325585 \\
\hline N20-0656 & 2020 Apr 20 & Human & Blood & $81 / F$ & SAMN15325586 \\
\hline N20-0662 & 2020 Apr 22 & Human & Blood & $86 / F$ & SAMN15325587 \\
\hline N20-0664 & 2020 Apr 22 & Human & Blood & $69 / F$ & SAMN15325588 \\
\hline N20-0674 & 2020 Apr 23 & Human & Blood & $84 / F$ & SAMN15325589 \\
\hline N20-689 & 2020 Apr 29 & Human & Blood & $63 / \mathrm{F}$ & SAMN15325590 \\
\hline N20-725 & 2020 May 4 & Human & Blood & $81 / \mathrm{M}$ & SAMN15325592 \\
\hline N20-737 & 2020 May 5 & Human & Blood & $86 / \mathrm{M}$ & SAMN15325593 \\
\hline N20-742 & 2020 May 6 & Human & Blood & $78 / \mathrm{F}$ & SAMN15325594 \\
\hline N20-743 & 2020 May 6 & Human & Blood & $37 / \mathrm{M}$ & SAMN15325595 \\
\hline N20-753 & 2020 May 8 & Human & Blood & $75 / \mathrm{M}$ & SAMN15325596 \\
\hline N20-754 & 2020 May 8 & Human & Blood & $85 / F$ & SAMN15325597 \\
\hline N20-771 & 2020 May 11 & Human & Blood & $95 / F$ & SAMN15325598 \\
\hline N20-778 & 2020 May 12 & Human & Blood & $95 / F$ & SAMN15325599 \\
\hline N20-851 & 2020 May 22 & Human & Perianal abscess & $85 / \mathrm{M}$ & SAMN15325600 \\
\hline N20-861 & 2020 May 26 & Human & Blood & $83 / F$ & SAMN15325601 \\
\hline N20-639 & 2020 Apr 30 & Food & Cheese sample & NA/NA & SAMN15325591 \\
\hline N20-757 & 2020 May 3 & Environment & Scrub sponge & NA/NA & SAMN15375881 \\
\hline N20-759 & 2020 May 3 & Environment & Drainage channel & NA/NA & SAMN15375882 \\
\hline N20-761 & 2020 May 3 & Environment & Door handle & NA/NA & SAMN15375884 \\
\hline N20-764 & 2020 May 3 & Environment & Cellar floor & NA/NA & SAMN15375885 \\
\hline N20-767 & 2020 May 3 & Environment & Ripening cellar floor & NA/NA & SAMN15375883 \\
\hline
\end{tabular}

\section{About the Author}

Dr. Nüesch-Inderbinen is a research associate at the Institute for Food Safety and Hygiene, University of Zurich, Zurich, Switzerland. Her primary research interest is pathogenic and antimicrobial-resistant bacteria in humans, animals, and the food chain.

\section{References}

1. Allerberger F, Wagner M. Listeriosis: a resurgent foodborne infection. Clin Microbiol Infect. 2010;16:16-23. https:/ / doi.org/ 10.1111/j.1469-0691.2009.03109.x

2. Buchanan RL, Gorris LG, Hayman MM, Jackson TC, Whiting RC. A review of Listeria monocytogenes: an update on outbreaks, virulence, dose-response, ecology, and risk assessments. Food Control. 2017;75:1-13. https:// doi.org/ 10.1016/j.foodcont.2016.12.016

3. Maury MM, Tsai YH, Charlier C, Touchon M, Chenal-Francisque $\mathrm{V}$, Leclercq A, et al. Uncovering Listeria monocytogenes hypervirulence by harnessing its biodiversity. Nat Genet. 2016;48:308-13. https:/ / doi.org/ 10.1038/ng.3501

4. Koopmans MM, Brouwer MC, Bijlsma MW, Bovenkerk S, Keijzers W, van der Ende A, et al. Listeria monocytogenes sequence type 6 and increased rate of unfavorable outcome in meningitis: epidemiologic cohort study. Clin Infect Dis. 2013;57:247-53. https://doi.org/10.1093/cid/cit250

5. European Centre for Disease Prevention and Control. Multi-country outbreak of Listeria monocytogenes PCR serogroup IVb MLST ST6, 2017 [cited 2020 Jun 6]. https://www.ecdc.europa.eu/en/publications-data/ rapid-risk-assessment-multi-country-outbreak-listeriamonocytogenes-pcr-serogroup

6. Althaus D, Jermini M, Giannini P, Martinetti G, Reinholz D, Nüesch-Inderbinen $\mathrm{M}$, et al. Local outbreak of Listeria monocytogenes serotype $4 \mathrm{~b}$ sequence type 6 due to contaminated meat pâté. Foodborne Pathog Dis. 2017;14:21922. https:// doi.org/10.1089/fpd.2016.2232

7. Thomas J, Govender N, McCarthy KM, Erasmus LK, Doyle TJ, Allam M, et al. Outbreak of listeriosis in South 
Africa associated with processed meat. N Engl J Med. 2020;382:632-43. https:// doi.org/10.1056/NEJMoa1907462

8. Smith AM, Tau NP, Smouse SL, Allam M, Ismail A, Ramalwa NR, et al. Outbreak of Listeria monocytogenes in South Africa, 2017-2018: laboratory activities and experiences associated with whole-genome sequencing analysis of isolates. Foodborne Pathog Dis. 2019;16:524-30. https://doi.org/10.1089/fpd.2018.2586

9. Halbedel S, Wilking H, Holzer A, Kleta S, Fischer MA, Lüth $S$, et al. Large nationwide outbreak of invasive listeriosis associated with blood sausage, Germany, 2018-2019. Emerg Infect Dis. 2020;26:1456-64. https:/ / doi.org/10.3201/ eid2607.200225

10. European Food Safety Authority. Trends and sources of zoonoses and zoonotic agents in foodstuffs, animals and feedingstuffs including information on foodborne outbreaks, antimicrobial resistance in zoonotic and indicator bacteria and some pathogenic microbiological agents in 2018 [cited 2020 Jun 6]. http:/ / www.efsa.europa.eu/sites/default/files/ zoocountryreport18ch.pdf

11. Ruppitsch W, Pietzka A, Prior K, Bletz S, Fernandez HL, Allerberger $\mathrm{F}$, et al. Defining and evaluating a core genome multilocus sequence typing scheme for whole-genome sequence-based typing of Listeria monocytogenes. J Clin Microbiol. 2015;53:2869-76. https://doi.org/10.1128/ JCM.01193-15

Address for correspondence: Magdalena Nüesch-Inderbinen, Institute for Food Safety and Hygiene, Vetsuisse Faculty, Winterthurerstrasse 272, University of Zurich, 8057 Zürich, Switzerland; e-mail: magdalena.nueesch-inderbinen@uzh.ch

\section{July 2020}

\section{Emerging Viruses}

- Case Manifestations and Public Health Response for Outbreak of Meningococcal W Disease, Central Australia, 2017

- Transmission of Chikungunya Virus in an Urban Slum, Brazil

- Public Health Role of Academic Medical Center in Community Outbreak of Hepatitis A, San Diego County, California, USA, 2016-2018

- Macrolide-Resistant Mycoplasma pneumoniae Infections in Pediatric Community-Acquired Pneumonia

- Efficient Surveillance of Plasmodium knowlesi Genetic Subpopulations, Malaysian Borneo, 2000-2018

- Bat and Lyssavirus Exposure among Humans in Area that Celebrates Bat Festival, Nigeria, 2010 and 2013

- Rickettsioses as Major Etiologies of Unrecognized Acute Febrile Illness, Sabah, East Malaysia

- Meningococcal W135 Disease Vaccination Intent, the Netherlands, 2018-2019

- Risk for Coccidioidomycosis among Hispanic Farm Workers, California, USA, 2018

- Paradoxal Trends in Azole-Resistant Aspergillus fumigatus in a National Multicenter Surveillance Program, the Netherlands, 2013-2018

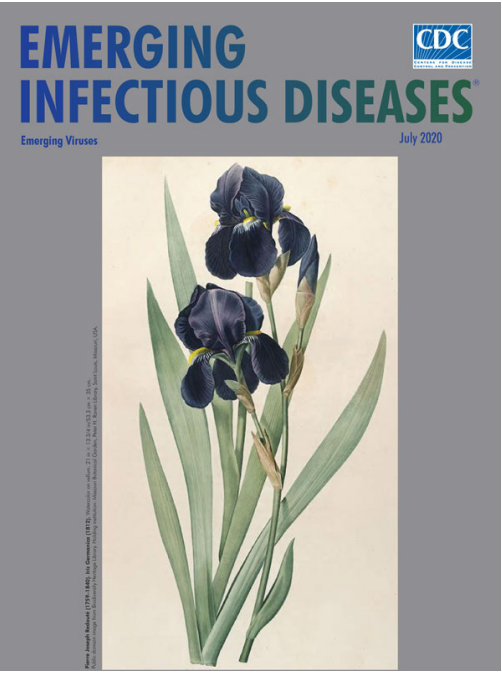

- Large Nationwide Outbreak of Invasive Listeriosis Associated with Blood Sausage, Germany, 2018-2019

- High Contagiousness and Rapid Spread of Severe Acute Respiratory Syndrome Coronavirus 2

- Identifying Locations with Possible Undetected Imported Severe Acute Respiratory Syndrome Coronavirus 2 Cases by Using Importation Predictions

- Atypical Manifestations of Cat-Scratch Disease, United States, 2005-2014
- Severe Acute Respiratory Syndrome Coronavirus 2-Specific Antibody Responses in Coronavirus Disease Patients

- Burden and Cost of Hospitalization for Respiratory Syncytial Virus in Young Children, Singapore

- Human Adenovirus Type 55 Distribution, Regional Persistence, and Genetic Variability

- Policy Decisions and Use of Information Technology to Fight COVID-19, Taiwan

- Sub-Saharan Africa and Eurasia Ancestry of Reassortant Highly Pathogenic Avian Influenza A(H5N8) Virus, Europe, December 2019

- Serologic Evidence of Severe Fever with Thrombocytopenia Syndrome Virus and Related Viruses in Pakistan

- Transmission of Legionnaires' Disease through Toilet Flushing

- Carbapenem Resistance Conferred by OXA-48 in K2-ST86 Hypervirulent Klebsiella pneumoniae, France

- Laboratory-Acquired Dengue Virus Infection, United States, 2018

- Linking Epidemiology and Whole-Genome Sequencing to Investigate Salmonella Outbreak, Massachusetts, USA, 2018

\section{EMERGING} INFECTIOUS DISEASES

To revisit the July 2020 issue, go to: https://wwwnc.cdc.gov/eid/articles/issue/26/7/table-of-contents 